\title{
Modeling the ecologic niche of plague in sylvan and domestic animal hosts to delineate sources of human exposure in the western United States
}

Michael Walsh, M.A. Haseeb

Plague has been established in the western United States (US) since 1900 following the west coast introduction of commensal rodents infected with Yersinia pestis via early industrial shipping. Over the last century, plague ecology has transitioned through cycles of widespread human transmission, urban domestic transmission among commensal rodents, and ultimately settled into the predominantly sylvan foci that remain today where it is maintained alternatively by enzootic and epizootic transmission. While zoonotic transmission to humans is much less common in modern times, significant plague risk remains in parts of the western US. Moreover, risk to some threatened species that are part of the epizootic cycle can be quite substantive. This investigation attempted to predict the risk of plague across the western US by modeling the ecologic niche of plague in sylvan and domestic animals identified between 2000 and 2015. A Maxent machine learning algorithm was used to predict this niche based on climate, altitude, land cover, and the presence of an important enzootic species, Peromyscus maniculatus. This model demonstrated good predictive ability (AUC $=86 \%$ ) and identified areas of high risk in central Colorado, north-central New Mexico, and southwestern and northeastern California. The presence of $P$. maniculatus, altitude, precipitation during the driest and wettest quarters, and distance to artificial surfaces, all contributed substantively to maximizing the gain function. These findings add to the known landscape epidemiology and infection ecology of plague in the western US and may suggest locations of particular risk to be targeted for wild and domestic animal intervention. 
1 Modeling the ecologic niche of plague in sylvan and domestic animal hosts to delineate sources of

2 human exposure in the western United States.

3 Michael G. Walsh ${ }^{1}$ and M.A. Haseeb ${ }^{1,2}$

4 1Department of Epidemiology and Biostatistics, School of Public Health, ${ }^{2}$ Departments of Cell Biology,

5 Pathology and Medicine, College of Medicine, State University of New York, Downstate Medical Center,

6 Brooklyn, New York, U.S.A.

Address correspondence to:

21 Michael Walsh, PhD, MPH

22 Assistant Professor, Epidemiology and Biostatistics

23 School of Public Health

24 State University of New York, Downstate

25450 Clarkson Avenue, Box 43

26 Brooklyn, NY 11203

27 thegowda@gmail.com, michael.walsh@downstate.edu

28 Phone: (347) 557-1108

29 
34

35

\section{Abstract}

Plague has been established in the western United States (US) since 1900 following the west coast introduction of commensal rodents infected with Yersinia pestis via early industrial shipping. Over the last century, plague ecology has transitioned through cycles of widespread human transmission, urban domestic transmission among commensal rodents, and ultimately settled into the predominantly sylvan foci that remain today where it is maintained alternatively by enzootic and epizootic transmission. While zoonotic transmission to humans is much less common in modern times, significant plague risk remains in parts of the western US. Moreover, risk to some threatened species that are part of the epizootic cycle can be quite substantive. This investigation attempted to predict the risk of plague across the western US by modeling the ecologic niche of plague in sylvan and domestic animals identified between 2000 and 2015. A Maxent machine learning algorithm was used to predict this niche based on climate, altitude, land cover, and the presence of an important enzootic species, Peromyscus maniculatus. This model demonstrated good predictive ability ( $A U C=86 \%$ ) and identified areas of high risk in central Colorado, north-central New Mexico, and southwestern and northeastern California. The presence of $P$. maniculatus, altitude, precipitation during the driest and wettest quarters, and distance to artificial surfaces, all contributed substantively to maximizing the gain function. These findings add to the known landscape epidemiology and infection ecology of plague in the western US and may suggest locations of particular risk to be targeted for wild and domestic animal intervention.

(1)

4

5

6


67

Introduction

Plague was introduced to North America in 1900 during the third major pandemic, which began in the $19^{\text {th }}$ century in China(Eskey and Haas 1940). Subsequently, the causative agent, Yersinia pestis, established in several foci across the semiarid regions of the western United States (US) where it is now maintained enzootically in large part by the deer mouse (Peromyscus maniculatus), with occasional epizootic events in some key species such as prairie dogs (Cynomys spp) and ground squirrels (Spermophilus spp.)(Eskey and Haas 1940; Gage and Kosoy 2005). Indeed, the geographic extent of the western US delineates one of the largest concentrations of sylvan plague foci in the modern world.

Despite the extensive foci of $Y$. pestis in the US, there are far fewer zoonotic transmissions to humans than occur in other geographic areas of significant plague foci, such as Madagascar or the Democratic Republic of the Congo(Stenseth et al. 2008). Nevertheless, the median annual incident cases of plague in the US was three (range 0 - 17) between 2001 and 2012(Kwit et al. 2015; Adams et al. 2014). Most of these human cases present as bubonic and can be quite severe, especially when not recognized and treated promptly with appropriate antibiotic therapy. As such, the ability to quantify the risk of zoonotic transmission from animals to humans across the region of established plague foci in the US remains a current research imperative. Flea bite and contact with infected animals accounted for $39.5 \%$ and $60.1 \%$, respectively, of all infection transmissions with known exposure history between 1965 and 2012(Kugeler et al. 2015). Moreover, while direct contact with wildlife is a recognized risk factor, plague is also often transmitted to domestic pets following exposure to wildlife, which then pass on the infection to their human caretakers(Craven et al. 1993). The importance of direct and indirect (via domestic animals) contact with wildlife notwithstanding, locations of animal plague in the landscape, which may act as conduits to zoonotic transmission of $Y$. pestis to humans, remain uncertain. Abiotic factors such as climate conditions and land cover and land use are of particular interest in understanding the distribution of animal plague. For example, relationships between animal plague occurrence and 
91 climate have been described in the western US, but in areas of more limited geographic extent(Holt et

92 al. 2009; Eisen, Glass, et al. 2007; Eisen, Reynolds, et al. 2007), while studies of land cover have focused

93 on sylvan host habitat without also considering proximity to developed land parcels(Eisen, Enscore, et

94 al. 2007). Equally important are biotic factors, such as the relative contribution of enzootic species (e.g.

95 P. maniculatus) to plague occurrence among epizootic species (e.g. Cynomys spp.) (Stenseth et al. 2008;

96 Gage and Kosoy 2005). Therefore, it would be useful to attempt to model the presence of animal plague

97 as a function of both abiotic and biotic features across the region. The current investigation sought to

98 predict the ecologic niche of epizootic plague based on the presence of plague in sylvan and domestic

99 animal hosts across all states west of the Mississippi River, and thereby identify spaces of potential

100 zoonotic plague transmission to humans. A machine learning approach was used to model the ecologic

101 niche as a function of climate, altitude, land cover, and the presence of the enzootic reservoir, $P$.

102 maniculatus.

103

Materials and Methods

104 Sixty-six reports of laboratory confirmed, geolocated infection with $Y$. pestis in sylvan and domestic

105 animal hosts were collected by the International Society of Infectious Diseases through the ProMED

106 electronic surveillance system between January 1, 2000 and August 31, 2015

107 (http://www.promedmail.org/). Geographic coordinates for each case were obtained using Google

108 Maps. These data, including the ProMED archive number for each report, are available via Figshare

109 (http://dx.doi.org/10.6084/m9.figshare.1538604). For inclusion in this study, the animal cases were

110 required to have been reported with a spatial resolution of 1 square kilometer or finer, meaning that

111 cases reported at $>1 \mathrm{~km}^{2}$ were of too coarse a resolution for consideration. In addition to the infections

112 in animal hosts, there were 48 laboratory-confirmed plague cases in humans over the same period.

113 However, these reports are generally only reported at the county level to protect patient privacy. 
114 Moreover, as many western US counties are large in area, the spatial resolution available for analysis

115 using these human cases would be quite coarse, so human cases were not included in these analyses.

116 The WorldClim Global Climate database was the source of all climate date used in this

117 investigation(WorldClim - Global Climate 2014). Annual mean temperature, annual mean precipitation,

118 mean temperature for the hottest and coldest quarters, mean precipitation for the wettest and driest

119 quarters, and isothermality from 1950 to 2000 were each extracted as 30 arc second resolution

120 rasters(Hijmans et al. 2005). Altitude was also extracted from this database at the same resolution. Each

121 pixel in these rasters represents the value of the measurement for that approximately $1 \mathrm{~km}^{2}$ area on the

122 Earth's surface.

123 A raster of the distribution of 9 unique land cover types was obtained from the Food and Agriculture

124 Organization of the United Nations' Global Land Cover - SHARE database(Latham et al. 2014). The

125 resolution of this raster was $1 \mathrm{~km}^{2}$. The land cover types represented in this raster were as follows: bare

126 soil, cropland, grassland, shrubland, sparse landscape, snow cover, tree cover, water, and artificial

127 surface. Each of the 9 land cover types was extracted from this raster and the distance between each

128 pixel and the nearest pixel of each unique land cover type was calculated to create a new distance

129 raster, of the same extent and resolution, for each land cover type. The distance calculations were

130 performed in the QGIS geographic information system (http://www.qgis.org/) using the proximity

131 function for calculating raster distances. The result of this process was a separate raster of $1 \mathrm{~km}^{2}$

132 resolution for each of the 9 land cover types described above wherein the value of each pixel is the

133 distance in meters between that pixel and the nearest pixel wherein the particular land cover feature is

134 present. This allows for modelling a spectrum of proximity to different land cover types in a given space

135 rather than the more crude approach of simply designating the space as present or absent for specific

136 land cover (see modelling description below). 
137 The Global Biodiversity Information Facility (GBIF) was used to identify the geographic distribution of

138 one of the most important reservoir species of $Y$. pestis in the enzootic transmission cycle, Peromyscus

139 maniculatus (http://www.gbif.org/). This database contained 94,983 field records of documented and

140 geolocated P. maniculatus individuals within the spatial extent of latitude $49.38436^{\circ} \mathrm{N}, 25.83738^{\circ} \mathrm{N}$, and

141 longitude $88.81702^{\circ} \mathrm{W}, 124.7631^{\circ} \mathrm{W}$. This extent delineates the boundaries of the western US within

142 which all previous plague occurrences, both animal and human, have been documented. This field

143 sample of 94,983 individual deer mice was used to create a raster of the predicted occurrence of the

144 reservoir across the western US using a Maxent model (see description of statistical methods below).

\section{Statistical Analysis}

146 A maximum entropy machine learning approach was employed to classify and map the ecologic niche of

147 plague infection in sylvan and domestic animal hosts. Because the aim of this exercise was to predict the

148 probability of animal plague occurrence across geographic space, machine learning is an attractive

149 analytic approach. Machine learning does not require the assumptions of a specific model form.

150 Algorithms are constructed to form decision trees, which partition a data space based on rules that

151 optimally identify homogeneity among predictors and a response (i.e. the presence of plague infected

152 animals)(Elith, Leathwick, and Hastie 2008). The analytic structure of these decisions trees is appealing

153 because they are robust to outliers, predictors may be of any form, and their hierarchical structure

154 inherently models interactions between the predictors. The Maxent machine learning algorithm, as

155 implemented in the dismo package in R(Hijmans et al. 2014), was used to predict plague in animal hosts

156 in the western United States. More specifically, the Maxent algorithm was used to predict the

157 probability that the biotic and abiotic conditions in a given location were suitable for the presence of

158 animal plague. This algorithm is based on a maximum entropy probability distribution, which holds that

159 in the absence of boundaries to species' dispersal, the distribution of those species will approach

160 uniformity. This algorithm is particularly appropriate for presence-only animal plague data because it 
161 does not require the unknown information associated with animal plague absences, which were not

162 available(Phillips, Anderson, and Schapire 2006; Franklin 2010). Documented species occurrences are

163 interpreted as presence points, while those points without documented occurrences are treated as the

164 background environment. In the Maxent model employed here, presence points are represented by the

165 locations of identified animal plague cases. As described above, the geographic extent of this analysis

166 was latitude $49.38436^{\circ} \mathrm{N}, 25.83738^{\circ} \mathrm{N}$, and longitude $88.81702^{\circ} \mathrm{W}, 124.7631^{\circ} \mathrm{W}$. Maxent models

167 consistently compare favorably with other approaches to machine learning, such as random forests or

168 support vector machines(Duan et al. 2014). The current analysis also compared the performance of

169 Maxent to predictive models derived from random forests and support vector machines to determine if

170 superior performance could be replicated in our study of animal plague.

171 For each $1 \mathrm{~km}^{2}$ geographic area within the spatial extent of the western US, the Maxent model was used

172 1) to predict the probability of habitat suitability of $P$. maniculatus, and 2) to predict the probability of

173 epizootic plague. The first model, targeting $P$. maniculatus, included mean annual temperature, mean

174 temperature during the coldest quarter, isothermality, mean precipitation during the driest quarter,

175 mean precipitation during the wettest quarter, distances to water, grassland, shrubland, trees, and

176 artificial surface as predictors of habitat suitability. The regularization parameter was set to 1.0 , to

177 balance between overly localized, overfit model predictions and overly generalized, broadly fit model

178 predictions. This output raster described the ecologic niche of $P$. maniculatus at a resolution of $1 \mathrm{~km}^{2}$.

179 Five-fold cross-validation was applied to the Maxent model, wherein the model was fit by first dividing

180 the training sets into $k=5$ subsets, and then cross-validating by iteratively fitting the model to 4 of the

181 combined subsets and testing against a 5th. This was repeated such that each of the $k=5$ subsets was

182 used as a test set during one iteration. The final cross-validated model was then evaluated against a test

183 dataset. The 5 subsets were comprised of 12,000 deer mouse observations each, with these being

184 randomly selected from the overall pool of training observations $(n=60,000$, or $\sim 2 / 3$ of the total 94,983 
185

deer mouse observations; see further description below). As a further evaluation of prediction error, the data were split into two subsets prior to beginning the analyses. The first subset was a training set $(n=$ $60,000)$, which was used to fit (or "train") the models, while the second subset $(n=34,983$ ) was left out of the model fitting process altogether and then used to test against the model predictions. The difference in model predictions based on training and testing data provided an assessment of the model's prediction error. The use of separate training and testing datasets reduces the typically high prediction error that attends overfitting of the data when all available data are used to train the model. In this analysis, the data were partitioned into $2 / 3$ and $1 / 3$ of the total observations for the training and test sets, respectively, and the area under the curve (AUC), reported as a percentage, was calculated to assess prediction error. The relative contribution is also reported for each predictor in the Maxent model. The relative contribution refers to the reduction of a loss function, which is referred to as the gain and is analogous to the residual deviance from generalized linear models, attributable to each predictor variable. The output raster of the predicted probability of $P$. maniculatus presence was then used as a predictor of epizootic plague. This subsequent Maxent model of epizootic plague included mean annual temperature, mean temperature during the coldest quarter, mean precipitation during the driest quarter, mean precipitation during the wettest quarter, distances to grassland, shrubland, cropland, sparse vegetation, bare soil, and artificial surface, and the predicted probability of $P$. maniculatus as predictors. The same 5 -fold cross validation process used to model $P$. maniculatus probability described above was used to model epizootic plague. The animal plague data $(n=66)$ were also partitioned into a training set comprising $2 / 3$ of the whole $(n=44)$ and a testing set comprising $1 / 3$ of the whole $(n=22)$. As above, the AUC was reported to assess the prediction error for the epizootic plague model and the relative contribution was reported for each predictor variable in the model. Variable response curves were also examined to more clearly elucidate specific relationships between individual variables and predicted animal plague occurrence. 
209 Model predictions of epizootic plague probability were converted to a binary score to designate

210 presence versus absence across a range of predicted plague occurrence. Four thresholds were thus

211 created at $25 \%, 50 \%, 75 \%$, and $90 \%$ predicted probability. These thresholds were used to create four

212 separate binary rasters designating each $1 \mathrm{~km}^{2}$ space as either present or absent for epizootic plague.

213 Results

214 The distribution of the $Y$. pestis infected animals as reported by the ProMED electronic surveillance

215 system of the ISID is presented in Figure 1. As expected, the distribution is limited to the western United

216 States, but with high concentrations of reported infected animals in Colorado, New Mexico, and

217 southern California. Central South Dakota marked the eastern boundary of reported animal plague

218 during the 15 year surveillance period, while north-central Montana delineated the northern boundary

219 in close proximity to the Canadian border(Hijmans et al. 2005).

Maps of the distribution of the environmental variables with the locations of infected animals overlaid

are presented in Figure 2. Some of the environmental features clearly demonstrate greater

heterogeneity than others. For example, precipitation, in both the driest and wettest quarters, and

temperature were unevenly distributed across the western states, as was proximity to artificial surface,

grassland, and bare soil land cover types. The map of predicted $P$. maniculatus in the first panel depicts

the probability surface of this enzootic species across the western US. The Maxent model used to

predict the species distribution identified mean annual temperature, isothermality, and distances to

artificial surfaces, grassland, and shrubland as the most influential with $34.7 \%, 22.6 \%, 14.9 \%$, $9 \%$, and

$8.9 \%$ relative contribution to the loss function, respectively. The AUC for the $P$. maniculatus species

distribution model was $76 \%$ indicating acceptable prediction error against test data.

230 The ecologic niche of epizootic plague as predicted by the Maxent model is presented in figures 3 and 4.

231 The former delineates a continuous risk surface as the probability of animal plague occurrence, while 
232 the latter figure depicts animal plague presence versus absence based on four thresholds of $25 \%, 50 \%$,

$23375 \%$, and $90 \%$ predicted probability of animal plague occurrence. In all maps counties are included to

234 provide a frame of reference within specific local municipalities. These maps highlight a pattern of

235 occurrence, albeit somewhat discontinuous, predicted along the eastern edge of the front range of the

236 American Rocky Mountains that extends from northwestern Montana down to north-central New

237 Mexico. Additional areas of high occurrence include southwestern and northeastern California,

238 southwestern South Dakota, central Arizona, as well as more localized areas in Oregon, Washington,

239 southern Idaho, and northern Utah. As the threshold for designating animal plague presence increased

240 from $25 \%$ to $90 \%$, the range of predicted presence narrowed until it was limited primarily to the front

241 range within the state of Colorado, with a few additional highly localized areas in Oregon, California, and

242 New Mexico. In the Maxent model used to predict the ecologic niche described in these maps, predicted

243 presence of P.maniculatus, altitude, distance to artificial surfaces, mean precipitation during the wettest

244 quarter, and mean precipitation during the driest quarter were the most influential environmental

245 variables contributing $50 \%, 17.2 \%, 8.3 \%, 7.2 \%$, and $7 \%$, respectively, to the loss function. Additionally,

246 mean annual temperature and distances to cropland, bare soil, and sparse vegetation modestly

247 contributed between $2 \%-3 \%$, while temperature during the coldest quarter and distances to grassland

248 and shrubland all contributed less than $1 \%$. The AUC derived from this Maxent model was $86 \%$, with

249 confidence bands based on $\mathrm{k}$-fold cross-validation replicates between $85.9 \%$ and $86.1 \%$, indicating

250 acceptable prediction error against the test data. Moreover, the Maxent model performed better than

251 either a random forests model ( $A \cup C=83 \%$ ) or a support vector machine model ( $A \cup C=70 \%$ ).

252 The variable response curves highlighted some interesting landscape features of habitat suitability for

253 animal plague (Supplemental Figure 1). The relationship between altitude and predicted animal plague

254 probability demonstrated a threshold at approximately 2000 meters, below which plague risk increased

255 linearly from sea level and after which no further increase in risk was observed. Thresholds were also 
256

257

258

259

260

261

262

263

264

265

266

267

268

269

270

271

272

273

274

275

276

277

278

noted for precipitation. Animal plague probability increased modestly with increasing mean precipitation during the driest quarter up to $50 \mathrm{~mm}$, while falling precipitously after this threshold until reaching 0 percent probability of plague by $100 \mathrm{~mm}$. Conversely, mean precipitation during the wettest quarter was associated with a sharp increase in predicted probability from $0 \mathrm{~mm}$ to $100 \mathrm{~mm}$ of precipitation, while steadily falling back down to zero between $100 \mathrm{~mm}$ and $1200 \mathrm{~mm}$ of precipitation. The sensitivity analysis, presented in Supplemental Figure 2, shows the probability of predicted plague occurrence at the $5 \mathrm{~km}^{2}$ predictor resolution to be very similar to that of the $1 \mathrm{~km}^{2}$ predictor resolution, including the same abiotic and biotic predictors in both models. Moreover, the AUC for this lower resolution model was $88 \%$. Therefore, this model of plague appears to be robust to scale.

Discussion

This investigation mapped the ecologic niche of epizootic plague across the western United States based on the presence of $Y$. pestis infection in sylvan and domestic animal hosts. The presence of $P$. maniculatus, mean precipitation during the driest and wettest quarters, altitude, and distance to artificial surfaces delineated influential landscapes with respect to the prediction of animal plague occurrence.

Plague foci in the western US have been recognized in sylvan species for several decades(Eskey and Haas 1940; Eisen, Enscore, et al. 2007). The transmission cycle of $Y$. pestis is defined by multi-directional, complex pathways that involve enzootic and epizootic transmission in animal hosts, and zoonotic and person-to-person transmission in humans(Stenseth et al. 2008; Gage and Kosoy 2005). The enzootic cycle involves vector-borne transmission of the pathogen between fleas and burrowing rodent species believed to be resistant to $Y$. pesits pathogenesis, particularly the deer mouse ( $P$. maniculatus) and the California vole (Microtus californicus)(Gage and Kosoy 2005; Poland, Quan, and Barnes 1994; S. . Quan and Kartman 1956; QUAN and KARTMAN 1962). The epizootic cycle emerges periodically in species 
279 highly susceptible to the pathogen and typically results in rapid and widespread transmission, and large

280 die-offs. The species most affected by these epizootics in the western US are prairie dogs (Cynomys

281 spp.), the black-footed ferret (Mustela nigripes), the California ground squirrel (Spermophilus beecheyi),

282 and the rock squirrel (Spermophilus variegatus)(Gage and Kosoy 2005; T. J. Quan et al. 1985; Poland,

283 Quan, and Barnes 1994). The enzootic and epizootic cycles are both maintained locally through flea-

284 borne transmission. However, more widespread diffusion beyond local sylvan populations is possible via

285 transport of fleas by predatory or passerine birds or via sylvan mammal predators or domestic cats that

286 prey on sylvan hosts [Stenseth, 2008; Gage and Kosoy, 2005; others]. Zoonotic transmission of bubonic

287 plague to humans is vector-borne or contact mediated. Vector-borne zoonotic transmission typically

288 results from fleas acquiring infection from commensal rodents (e.g. Rattus norvegicus) and then passing

289 the infection on to humans following the death of the commensal, while contact transmission requires

290 contact with an infected animal, either domestic or sylvan (often by handling the dead carcass in the

291 case of sylvan hosts)(Stenseth et al. 2008; Gage and Kosoy 2005). Domestic cats are the primary source

292 of domestic zoonoses of plague as they 1) often prey on wildlife hosts, and 2) are highly susceptible to $Y$.

293 pestis infection and pathogenesis. Interestingly, pneumonic plague can also be transmitted from

294 domestic cats to humans, but is more commonly transmitted person-to-person. These transmission

295 cycles of plague ecology reveal several animal hosts that may be relevant for zoonotic transmission to

296 humans, but the emergence of epizootics is generally believed to drive spillover to humans(Gage and

297 Kosoy 2005; Kugeler et al. 2015). As such, species involved with the epizootic transmission cycle were

298 the current focus in attempting to identify areas of high animal plague occurrence in the landscape,

299 which may have the potential drive plague spillover to humans. The current study examined plague in

300 animals relevant to this epizootic cycle (squirrels, prairie dogs, and sylvan predators), or those domestic

301 animals (primarily cats) that may acquire the infection via their intersection with the epizootic cycle and

302 which may then pass on the infection to their human caretakers. Plague spillover to humans via the 
303

304

305

306

307

308

309

310

311

312

313

314

315

316

317

318

319

320

321

322

323

324

325

326

enzootic cycle is believed to be a rare occurrence and so the relevance of species within this cycle to the human risk surface is probably minimal(Gage and Kosoy 2005). Nevertheless, species in this cycle may play a role in the force of infection for highly susceptible species in the epizootic cycle and, therefore, the ecologic niche of one such species (P. maniculatus) was used to predict the presence of epizootic plague. While we did not assess the force of infection directly in this this study, our findings did show that $P$. maniculatus presence was the greatest contributor to the probability of animal plague occurrence in highly susceptible animals.

Altitude has been previously identified as an important feature of the landscape epidemiology of plague in the western US(Eisen, Reynolds, et al. 2007; Eisen, Enscore, et al. 2007) and in other parts of the Americas(Schneider et al. 2014). While increasing elevation has been associated with a greater occurrence of plague, most investigations have identified an elevation ceiling, beyond which plague occurrence either no longer increases, or reverses and begins to decrease(Eisen, Enscore, et al. 2007;

Eisen, Reynolds, et al. 2007; Schneider et al. 2014). Indeed, a similar threshold was demonstrated in the current study. Increasing elevation was linearly associated with increasing probability of animal plague until a threshold was reached at 2000 meters, after which the probability of occurrence reached a plateau. The reason for such a threshold is not entirely clear, but may coincide with generally increasingly favorable habitat conditions with increasing altitude from sea level ultimately giving way subsequently to more limited distribution of relevant habitat and host species in areas of the highest altitudes. For example, certain tree species such as pinyon pines or juniper pines are preferred by $P$. maniculatus in southwestern landscapes, which occur within a range of moderate elevation and which may influence the occurrence of epizootic animal plague via the distribution of an important enzootic reservoir(Eisen, Glass, et al. 2007; Eisen, Enscore, et al. 2007).

As with altitude, climate has been previously shown to be an important predictor of both sylvan plague and zoonotic transmission to humans(Eisen, Glass, et al. 2007; Enscore et al. 2002; Holt et al. 2009; 
327 Parmenter et al. 1999). These studies have generally found that increasing precipitation during the

328 winter and spring months were associated with increasing incidence of plague in the following summer.

329 In the current study, increasing mean precipitation during the wettest quarter was associated with

330 increasing plague risk up to a threshold of $100 \mathrm{~mm}$ in a three month period. After this threshold,

331 however, plague risk begins to steadily decline in a linear fashion with increasing mean precipitation

332 during the wettest period. Increasing mean precipitation during the driest quarter also corresponded to

333 a more moderate increase in plague risk up to a threshold of $50 \mathrm{~mm}$, after which plague risk dropped

334 very quickly back to zero. Similar precipitation patterns were observed previously in California(Holt et al.

335 2009) and New Mexico(Parmenter et al. 1999). This may suggest that the importance of precipitation to

336 plague risk is seasonal with more rain during the wet period perhaps influencing population dynamics in

337 sylvan host species and subsequent plague transmission cycles. In particular, periods of higher

338 precipitation may correspond to subsequent periods of greater food abundance, which may increase the

339 range and volume of important enzootic species or epizootic species. This would be expected to be

340 particularly relevant for $P$. maniculatus in its favored pinyon-juniper woodlands in the southwest due to

341 increased production of pinyon seeds. Parmenter et al. showed that increasing precipitation was an

342 important precursor to emergent human infections, but not epizootics as such(Parmenter et al. 1999).

343 Moreover, their findings only held at highly localized, large scales of analysis rather than across broad

344 geographic context.

345 Proximity to artificial surfaces was associated with increasing animal plague occurrence. Moreover, this

346 was the most influential land cover type (relative contribution $=8.3 \%$ ), as compared with grassland,

347 cropland, shrubland, and bare soil. To the best of the authors' knowledge, this is the first study to

348 demonstrate an influence of developed land on animal plague occurrence in the US. Landscape

349 fragmentation has previously been shown to be associated with animal and human plague occurrence in

350 other parts of the world with significant plague foci, such as Madagascar(Duplantier et al.) and 
351 Tanzania(McCauley et al. 2015). Increasingly developed landscapes encroach on natural habitat and

352 usher changes that may increase contact with sylvan species, which in turn may expand previously

353 closed or limited transmission cycles and lead to zoonoses(Daszak, Cunningham, and Hyatt 2000;

354 Estrada-Peña et al. 2014; Wolfe, Dunavan, and Diamond 2007; Woolhouse and Gowtage-sequeria 2005).

355 While the current investigation did not measure fragmentation directly, the distribution of developed

356 land cover, as measured by artificial surfaces, may serve as a proxy for fragmentation and, thus, provide

357 initial support for the role of human pressure in the infection ecology and landscape epidemiology of

358 plague in the western US.

359 It is important to recognize several limitations inherent in the current study. First, the rasters for

360 temperature and precipitation consisted of single composite measures over the period 1950 to 2000.

361 Given that these rasters were averaged over a 50 year time span, the temporal resolution was coarse

362 even though the spatial resolution was fine $\left(\sim 1 \mathrm{~km}^{2}\right)$. Nevertheless, the measures of temperature and

363 precipitation in this study are believed to be sound assessments of the general climate of the western

364 US, and thus provide a robust, if somewhat conservative and temporally coarse, approach to quantifying

365 background climate. Second, this study relies on reports that are archived in the ProMED surveillance

366 system and, thus, may have missed animal infections of $Y$. pestis not reported by local public health

367 departments or by land and wildlife management authorities. Third, the number of occurrences of

368 animal plague reported between 2000 and 2015 is relatively small, providing a sample size of only 66

369 precisely geolocated animal cases. Therefore, this collection of sylvan and domestic animal infections

370 may not be representative of the total potential events in the western US. Nevertheless, this analysis

371 was thorough in validating the sparse data by 1) using 5 -fold cross-validation 2) validating the training

372 models against test data, which showed low prediction error $(A \cup C=86 \%)$, and 3 ) analyzing the

373 landscape variables at 2 different resolutions $\left(1 \mathrm{~km}^{2}\right.$ and $\left.5 \mathrm{~km}^{2}\right)$ to show consistency of the predictions

374 across scale. Furthermore, the Maxent model is robust to spatial sampling bias that may be inherent in 
375

376

presence-only species distribution data. Most importantly, we do not purport these data to represent the definitive picture of animal plague presence in the western US. Rather, these findings are recognized as tentative but, nevertheless, useful to the evolving understanding of the landscape epidemiology and infection ecology of plague in sylvan and domestic animals in the western US. It is equally recognized that this model of plague risk will require the scrutiny of ongoing validation as more data become available. More specifically, direct measurement of animals in the specific locations of distinct enzootic and epizootic transmission cycles must be made, particularly with a view toward interactions between these cycles and subsequent zoonotic transmission to humans. This endeavor will require broad field sampling to record animal serology, habitat description at a much finer resolution and richer detail (i.e. describing not simply land cover, but providing more precise descriptions of habitat parcels in terms of biotic and abiotic properties), specific occupational and social practices, and residential circumstances, that bring humans into close proximity to animal hosts. Such research will be expensive, but it is necessary if we are to generate rich data suited to inform wildlife and domestic interventions that can limit dangerous human-animal encounters and block zoonotic plague transmission. Localized field investigations into climate and altitude have been conducted in the western US and generally support the findings from the current study, as described above. However, these results corroborate, but do not validate, the current findings. More extensive field studies conducted within the same geographic extent of the western US will be required to validate our predictions. In particular, areas designated to have a particularly high probability of animal plague occurrence (e.g. $75 \%$ - $90 \%$ ) may be targeted by local health departments and wildlife management authorities for animal trapping and serologic testing. The predicted high risk areas could then be compared to adjacent areas predicted to have a low probability of occurrence. 
400 This investigation predicted animal plague occurrence across the western US based on reported

401 occurrences of plague in sylvan and domestic animal hosts. The distribution of $P$. maniculatus, presumed

402 to be an important species in the enzootic cycle of plague, altitude, precipitation during both the driest

403 and wettest quarters, and proximity to developed land parcels were identified as important features of

404 habitat suitability for animal plague, and may demarcate potential areas of zoonotic transmission to

405 humans.

406

407

408

409

410

411

412

413

414

415

416

417

418

419

420

421

422

423 Acknowledgements: None.

424

425 


\section{References}

Adams, Deborah A, Ruth Ann Jajosky, Umed Ajani, Jeffrey Kriseman, Pearl Sharp, Diana H Onwen, Alan W Schley, et al. 2014. "Summary of Notifiable Diseases--United States, 2012." MMWR. Morbidity and Mortality Weekly Report 61 (53): 1-121. http://www.ncbi.nlm.nih.gov/pubmed/25233134.

Craven, R B, G O Maupin, M L Beard, T J Quan, and A M Barnes. 1993. "Reported Cases of Human Plague Infections in the United States, 1970-1991." Journal of Medical Entomology 30 (4): 758-61. http://www.ncbi.nlm.nih.gov/pubmed/8395603.

Daszak, P, A A Cunningham, and A D Hyatt. 2000. “Emerging Infectious Diseases of Wildlife--Threats to Biodiversity and Human Health." Science (New York, N.Y.) 287 (5452): 443-49. http://www.ncbi.nlm.nih.gov/pubmed/10642539.

Duan, Ren-Yan, Xiao-Quan Kong, Min-Yi Huang, Wei-Yi Fan, and Zhi-Gao Wang. 2014. "The Predictive Performance and Stability of Six Species Distribution Models." PloS One 9 (11): e112764. doi:10.1371/journal.pone.0112764.

Duplantier, Jean-Marc, Jean-Bernard Duchemin, Suzanne Chanteau, and Elisabeth Carniel. "From the Recent Lessons of the Malagasy Foci towards a Global Understanding of the Factors Involved in Plague Reemergence." Veterinary Research 36 (3): 437-53. doi:10.1051/vetres:2005007.

Eisen, Rebecca J, Russell E Enscore, Brad J Biggerstaff, Pamela J Reynolds, Paul Ettestad, Ted Brown, John Pape, et al. 2007. "Human Plague in the Southwestern United States, 1957-2004: Spatial Models of Elevated Risk of Human Exposure to Yersinia Pestis." Journal of Medical Entomology 44 (3): 53037. http://www.ncbi.nlm.nih.gov/pubmed/17547242.

Eisen, Rebecca J, Gregory E Glass, Lars Eisen, James Cheek, Russell E Enscore, Paul Ettestad, and Kenneth L Gage. 2007. "A Spatial Model of Shared Risk for Plague and Hantavirus Pulmonary Syndrome in the Southwestern United States." The American Journal of Tropical Medicine and Hygiene 77 (6): 999-1004. http://www.ncbi.nlm.nih.gov/pubmed/18165511.

Eisen, Rebecca J, Pamela J Reynolds, Paul Ettestad, Ted Brown, Russell E Enscore, Brad J Biggerstaff, James Cheek, et al. 2007. "Residence-Linked Human Plague in New Mexico: A Habitat-Suitability Model." The American Journal of Tropical Medicine and Hygiene 77 (1): 121-25. http://www.ncbi.nlm.nih.gov/pubmed/17620642.

Elith, J, J R Leathwick, and T Hastie. 2008. "A Working Guide to Boosted Regression Trees." The Journal of Animal Ecology 77 (4): 802-13. doi:10.1111/j.1365-2656.2008.01390.x.

Enscore, Russell E, Brad J Biggerstaff, Ted L Brown, Ralph E Fulgham, Pamela J Reynolds, David M Engelthaler, Craig E Levy, et al. 2002. "Modeling Relationships between Climate and the Frequency of Human Plague Cases in the Southwestern United States, 1960-1997." The American Journal of Tropical Medicine and Hygiene 66 (2): 186-96. http://www.ncbi.nlm.nih.gov/pubmed/12135292.

Eskey, Clifford Rush, and Victor Howard Haas. 1940. Plague in the Western Part of the United States. U.S. Government Printing Office. https://books.google.com/books?id=5cAdKAAACAAJ\&pgis=1. 
462

463

464

465

466

467

468

469

470

471

472

473

474

475

476

477

478

479

480

481

482

483

484

485

486

487

488

489

490

491

492

493

494

495

496

497

Estrada-Peña, Agustín, Richard S Ostfeld, a Townsend Peterson, Robert Poulin, and José de la Fuente. 2014. "Effects of Environmental Change on Zoonotic Disease Risk: An Ecological Primer." Trends in Parasitology 30 (4): 205-14. doi:10.1016/j.pt.2014.02.003.

Franklin, Janet. 2010. Mapping Species Distributions: Spatial Inference and Prediction. First. Cambridge: Cambridge University Press. https://books.google.com/books?id=CkshAwAAQBAJ\&pgis=1.

Gage, Kenneth L, and Michael Y Kosoy. 2005. "Natural History of Plague: Perspectives from More than a Century of Research." Annual Review of Entomology 50 (January): 505-28. doi:10.1146/annurev.ento.50.071803.130337.

Hijmans, Robert J., Susan E. Cameron, Juan L. Parra, Peter G. Jones, and Andy Jarvis. 2005. "Very High Resolution Interpolated Climate Surfaces for Global Land Areas." International Journal of Climatology 25 (15): 1965-78. doi:10.1002/joc.1276.

Hijmans, Robert J., Steven Phillips, J R Leathwick, and J Elith. 2014. "Package 'Dismo.'” The Comprehensive R Archive Network. http://cran.r-project.org/web/packages/dismo/dismo.pdf.

Holt, Ashley C, Daniel J Salkeld, Curtis L Fritz, James R Tucker, and Peng Gong. 2009. "Spatial Analysis of Plague in California: Niche Modeling Predictions of the Current Distribution and Potential Response to Climate Change." International Journal of Health Geographics 8 (January): 38. doi:10.1186/1476072X-8-38.

Kugeler, Kiersten J, J Erin Staples, Alison F Hinckley, Kenneth L Gage, and Paul S Mead. 2015. "Epidemiology of Human Plague in the United States, 1900-2012." Emerging Infectious Diseases 21 (1): 16-22. doi:10.3201/eid2101.140564.

Kwit, Natalie, Christina Nelson, Kiersten Kugeler, Jeannine Petersen, Lydia Plante, Hayley Yaglom, Vicki Kramer, et al. 2015. "Human Plague - United States, 2015." MMWR. Morbidity and Mortality Weekly Report 64 (33): 918-19. http://www.ncbi.nlm.nih.gov/pubmed/26313475.

Latham, John, Renato Cumani, Ilaria Rosati, and Mario Bloise. 2014. "FAO Global Land Cover SHARE Beta-Release 1.0 Database." Food and Agriculture Organization of the United Nations, Land and Water Division.

McCauley, Douglas J, Daniel J Salkeld, Hillary S Young, Rhodes Makundi, Rodolfo Dirzo, Ralph P Eckerlin, Eric F Lambin, Lynne Gaffikin, Michele Barry, and Kristofer M Helgen. 2015. "Effects of Land Use on Plague (Yersinia Pestis) Activity in Rodents in Tanzania." The American Journal of Tropical Medicine and Hygiene 92 (4): 776-83. doi:10.4269/ajtmh.14-0504.

Parmenter, R R, E P Yadav, C A Parmenter, P Ettestad, and K L Gage. 1999. "Incidence of Plague Associated with Increased Winter-Spring Precipitation in New Mexico." The American Journal of Tropical Medicine and Hygiene 61 (5): 814-21. http://www.ncbi.nlm.nih.gov/pubmed/10586917.

Phillips, Steven J., Robert P. Anderson, and Robert E. Schapire. 2006. "Maximum Entropy Modeling of Species Geographic Distributions." Ecological Modelling 190 (3-4): 231-59.

doi:10.1016/j.ecolmodel.2005.03.026. 
498

499

500

501

502

503

504

505

506

507

508

509

510

511

512

513

514

515

516

517

518

519

520

521

522

523

524

525

526

Poland, Jack D., T.J. Quan, and Allen M. Barnes. 1994. "Handbook of Zoonoses, Second Edition: Bacterial, Rickettsial, Chlamydial, and Mycotic Zoonoses." In , 93-112. Boca Raton: CRC Press. https://books.google.com/books?id=wcE1AR_CLy8C\&pgis=1.

QUAN, S F, and L KARTMAN. 1962. "Ecological Studies of Wild Rodent Plague in the San Francisco Bay Area of California. VIII. Susceptibility of Wild Rodents to Experimental Plague Infection." Zoonoses Research 1 (January): 121-44. http://www.ncbi.nlm.nih.gov/pubmed/14489383.

Quan, S.F, and Leo Kartman. 1956. "The Resistance of Microtus and Peromyscus to Infection by Pasteurella Pestis." Transactions of the Royal Society of Tropical Medicine and Hygiene 50 (1): $104-$ 5. doi:10.1016/0035-9203(56)90017-7.

Quan, T J, A M Barnes, L G Carter, and K R Tsuchiya. 1985. "Experimental Plague in Rock Squirrels, Spermophilus Variegatus (Erxleben)." Journal of Wildlife Diseases 21 (3): 205-10. http://www.ncbi.nlm.nih.gov/pubmed/4032620.

Schneider, Maria Cristina, Patricia Najera, Sylvain Aldighieri, Deise I Galan, Eric Bertherat, Alfonso Ruiz, Elsy Dumit, Jean Marc Gabastou, and Marcos A Espinal. 2014. "Where Does Human Plague Still Persist in Latin America?" PLoS Neglected Tropical Diseases 8 (2): e2680. doi:10.1371/journal.pntd.0002680.

Stenseth, Nils Chr, Bakyt B Atshabar, Mike Begon, Steven R Belmain, Eric Bertherat, Elisabeth Carniel, Kenneth L Gage, Herwig Leirs, and Lila Rahalison. 2008. "Plague: Past, Present, and Future." PLoS Medicine 5 (1): e3. doi:10.1371/journal.pmed.0050003.

Wolfe, Nathan D, Claire Panosian Dunavan, and Jared Diamond. 2007. “Origins of Major Human Infectious Diseases." Nature 447 (7142): 279-83. doi:10.1038/nature05775.

Woolhouse, Mark E J, and Sonya Gowtage-sequeria. 2005. "Host Range and Emerging and Reemerging Pathogens" 11 (12).

WorldClim - Global Climate. 2014. "Data for Current Conditions ( 1950-2000) | WorldClim - Global Climate Data." WorldClim - Global Climate Data. Accessed October 23. http://www.worldclim.org/current. 
1

The distribution of the 66 laboratory-confirmed animal plague cases identified through the ProMED system between January 1, 2000 and August 31, 2015 in the United States.

Map data: Google, @2015 TerraMetrics. 


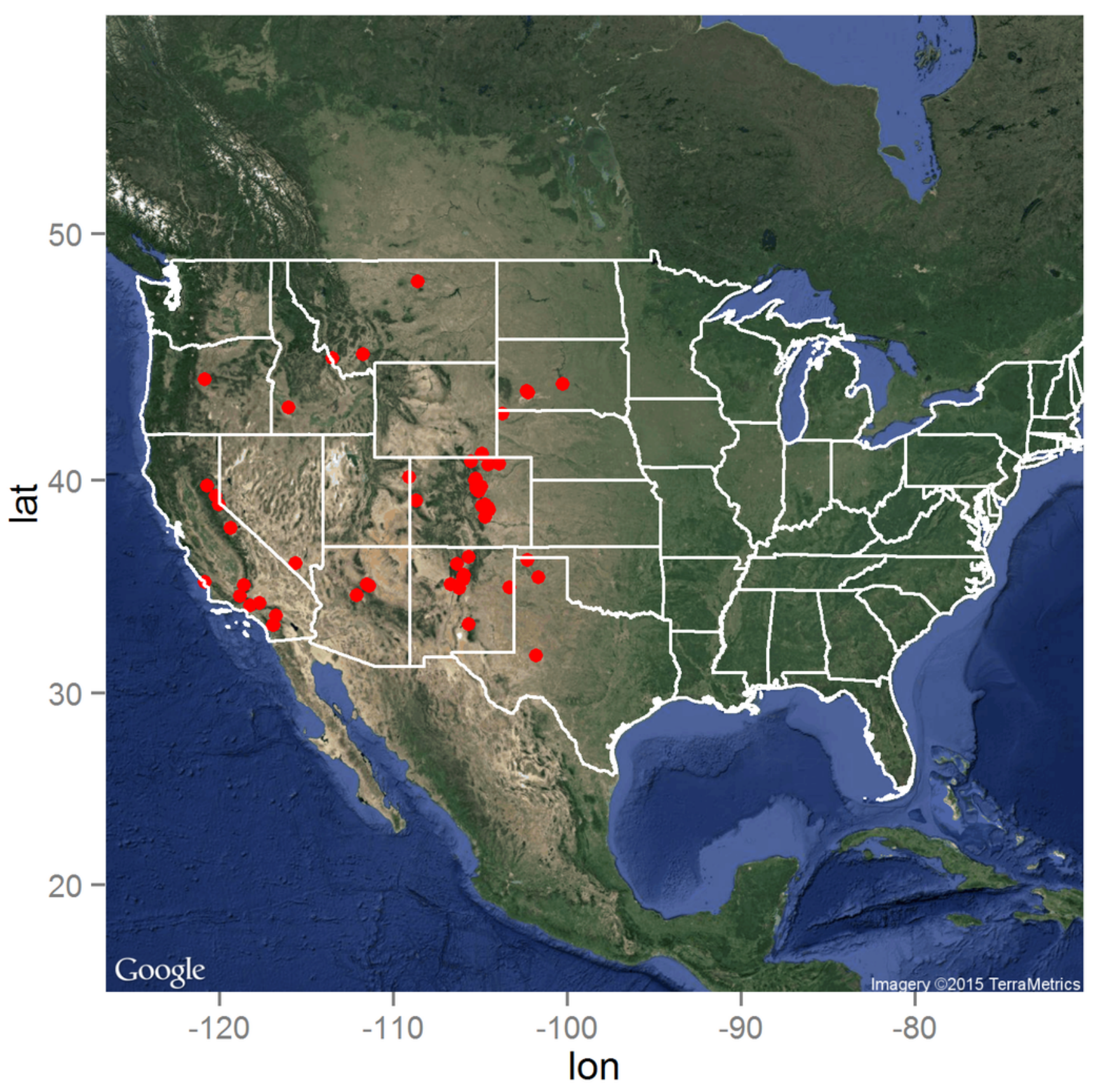


2

Rasters of model predictors.

The predicted distribution of Peromyscus maniculatus (deer mouse), altitude, mean precipitation during the driest and wettest quarters, distances to land cover types, and mean annual temperature with the distribution of the 66 animal plague cases overlaid (dots).
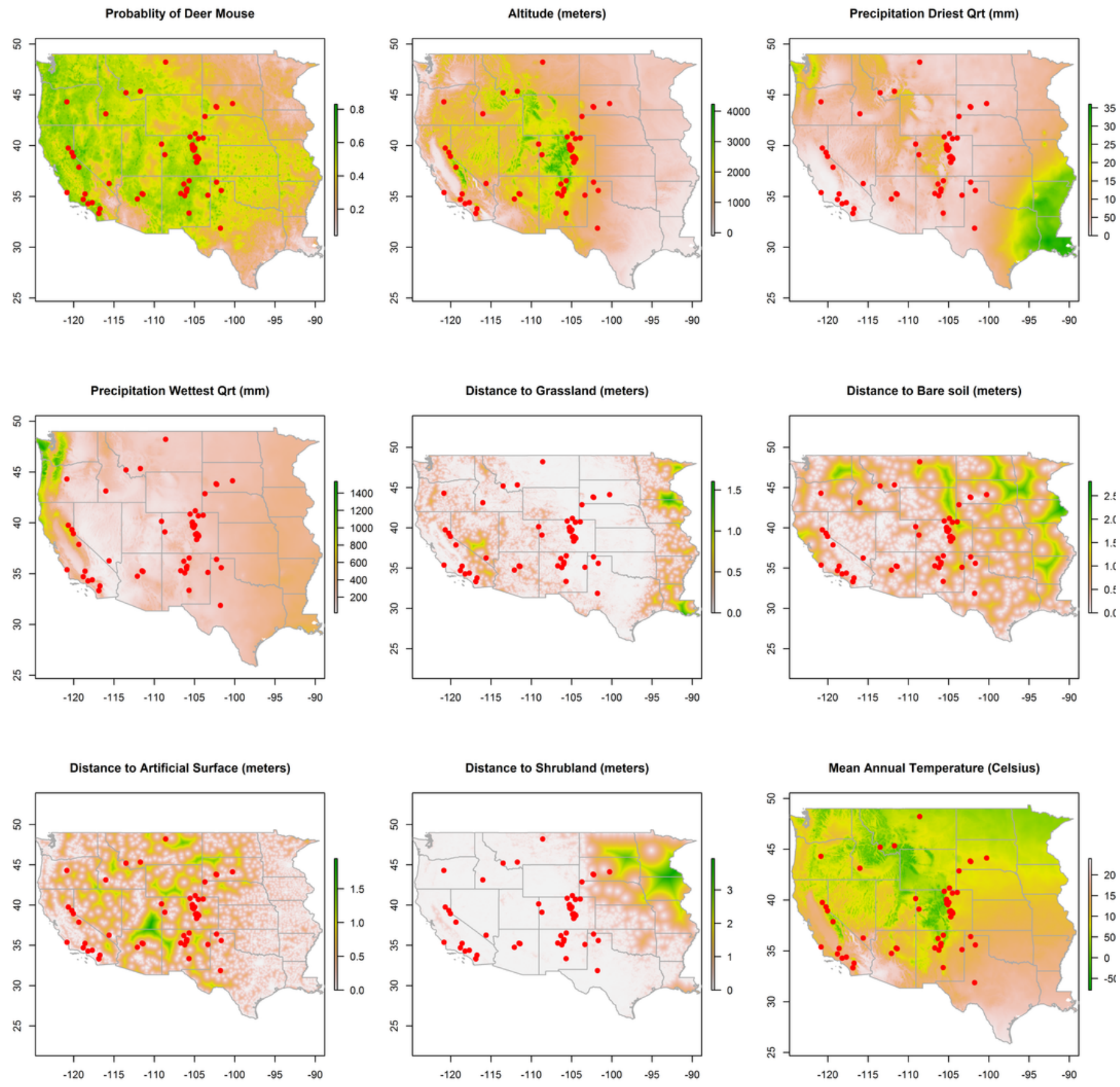
3

Predicted probability of epizootic animal plague.

These risk surfaces are based on the ecologic niche of animal plague as derived from the Maxent model.

\section{Probabililty of Plague}

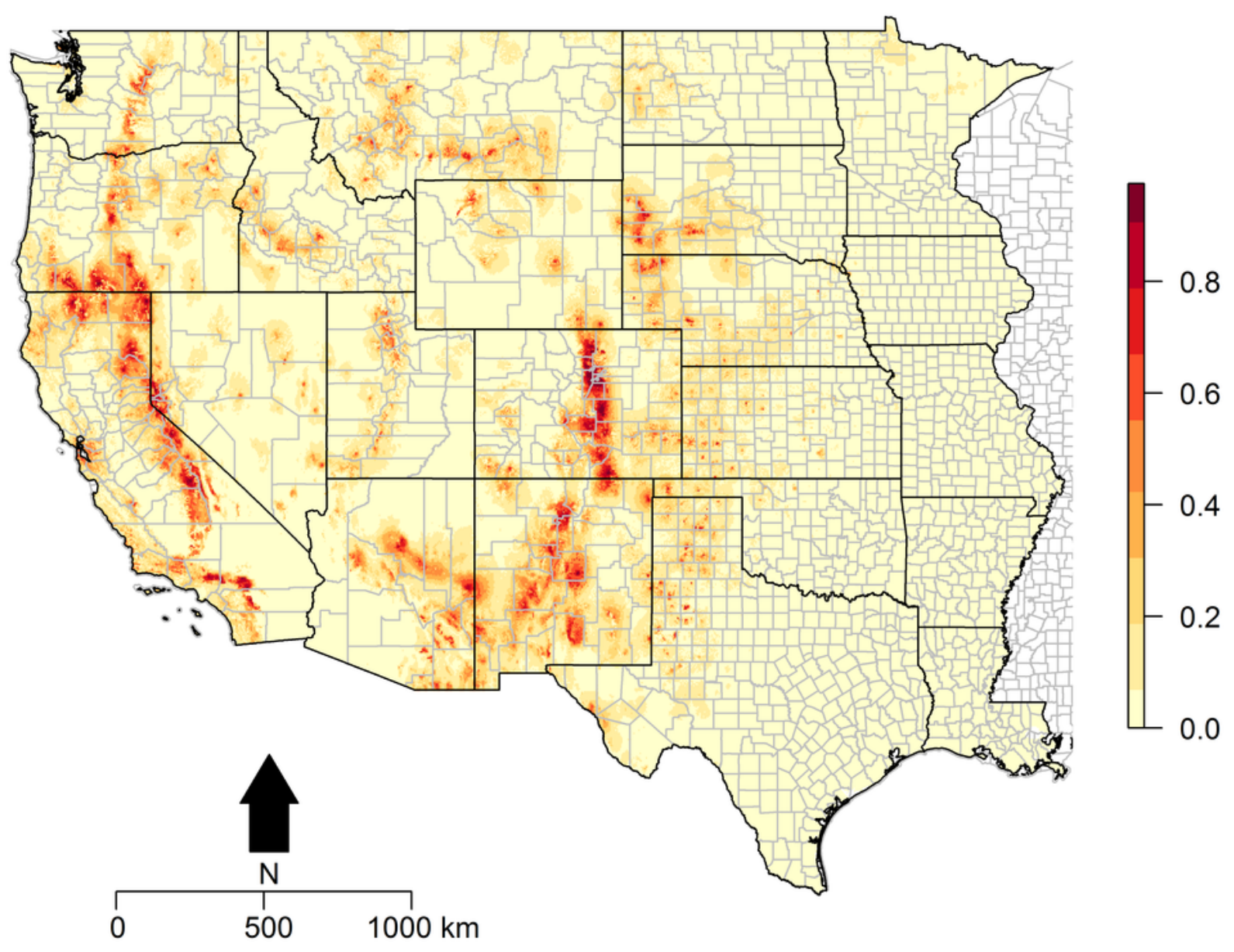


4

Predicted presence of animal plague based on thresholds of $25 \%, 50 \%, 75 \%$, and $90 \%$ probability of animal plague occurrence derived from the Maxent model.

Epizotic plague presence at $\mathbf{2 5 \%}$ or greater predicted probability

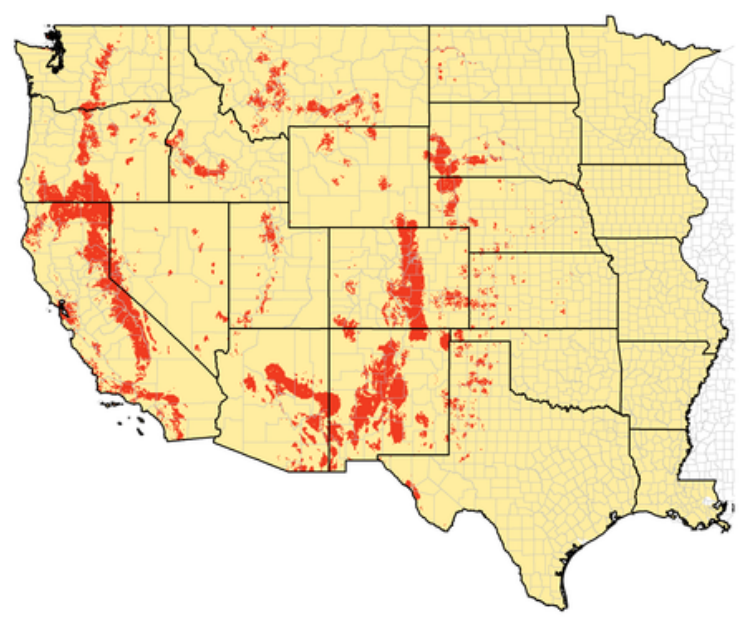

Epizotic plague presence at $\mathbf{7 5 \%}$ or greater predicted probability

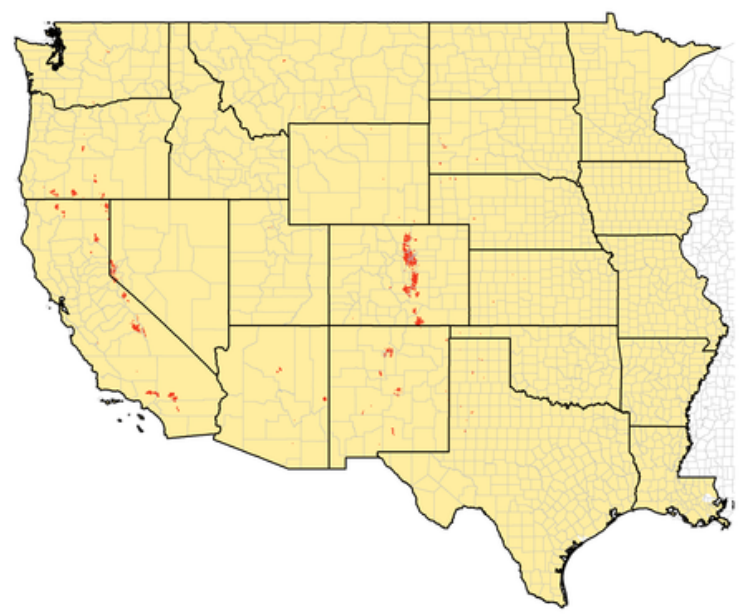

Epizotic plague presence at $\mathbf{5 0} \%$ or greater predicted probability

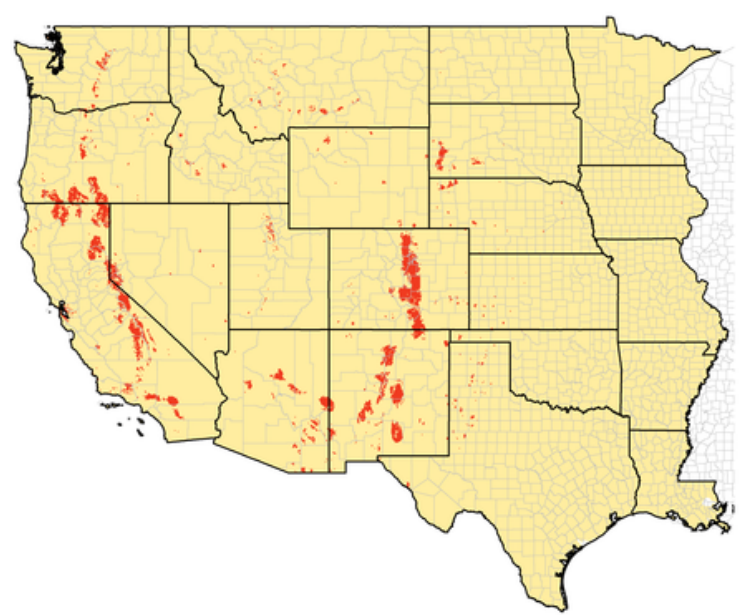

Epizotic plague presence at $90 \%$ or greater predicted probability

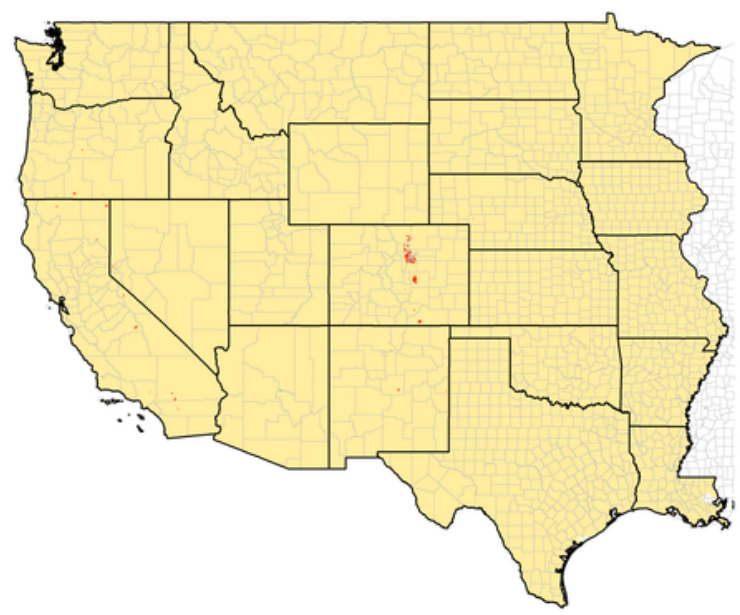

